Amerika muss bereit sein, das Wesen Europas zu verstehen und damit seine Modernität zu begreifen. Es muss in seinem eigensten Interesse bereit sein, Europa nicht nur als militärischen oder politischen Verbündeten oder als wirtschaftlichen Mitstreiter zu sehen, sondern als echten Partner mit dem ihm eigenen Charakter. Die Amerikaner werden dies verstehen, wenn sie es verstehen müssen, und wenn wir Europäer mehr in die Waagschale zu werfen bereit sind.

Die USA sind der mächtigste Akteur in der heutigen Welt Europa ist der modernste. »Europa ist ein Beitrag zu einer besseren Welt.« Nichts ist vordringlicher, als dieses Wort Jean Monnets stärker - sehr viel stärker - zur Geltung zu bringen.

\title{
Kurs halten unter schweren Bedingungen: Bilanz und Perspektiven grüner Sicherheits- und Friedenspolitik
}

\author{
Winfried Nachtwei, MdB, Bünd- \\ nis 90/Die Grünen, Mitglied \\ des Verteidigungsausschusses \\ und des Unterausschusses $A b$ - \\ rüstung, Rüstungskontrolle und \\ Nichtverbreitung, Berlin
}

»Deutsche Außenpolitik ist Friedenspolitik « behaupteten SPD und Bündnis 90/Die Grünen im Oktober 1998 in ihrer Koalitionsvereinbarung voller friedenspolitischer Selbstgewissheit. Sie versprachen, »sich mit aller Kraft um die Entwicklung und Anwendung von wirksamen Strategien und Instrumenten der Krisenprävention und der friedlichen Konfliktregelung zu bemühen« und dafür eine Infrastruktur aufzubauen.

Vier Jahre später kann man festhalten, dass sich Deutschland unter Rot-Grün in der Tat zu einem international anerkannten und vielfach treibenden Friedensakteur entwickelt hat. Wenn es darum ging, Handlungsspielräume friedensförderlich $\mathrm{zu}$ nutzen, hat der grüne Faktor sicher eine weit über die 6,7\% hinausragende Rolle gespielt. Doch während im Ausland die Leistungen der deutschen Außen- und Friedenspolitik positiv gewürdigt werden, scheiden sich daran in Deutschland die Geister. Unter Rot-Grün beteiligte sich das demokratische Deutschland erstmalig an zwei Kriegseinsätzen. Die Auslandseinsätze der Bundeswehr wuchsen in Dimensionen, wie sie 1998 kaum jemand für möglich gehalten hätte.

Kein Wunder, dass gerade bei Friedensbewegten, bei Teilen der Friedensforschung und der rot-grünen Anhängerschaft die Enttäuschung tief sitzt und die Urteile hart sind. Rot-Grün habe die Kultur der militärischen Zurückhaltung aufgegeben und das Militär zum normalen Instrument deutscher Außenpolitik gemacht, ja, Rot-Grün treibe vor allem Kriegspolitik im machtpolitischen Interesse. Vor allem den Grünen hängt dabei der Vorwurf an, ihre pazifistischen Prinzipien verraten zu haben.

Auf der anderen Seite wirft die bürgerliche Opposition Rot-Grün vor, die Bundeswehr kaputt zu sparen und damit die internationale Position der Bundesrepublik, ja, ihre Sicherheit zu gefährden.
Jenseits dieser Pole lautstarker Kritik findet rot-grüne Außenund Sicherheitspolitik zugleich breite Zustimmung über Parteigrenzen hinweg. Ihr wird zugute gehalten, in verantwortlicher Weise und als verlässlicher Partner zur internationalen Sicherheit beizutragen. Die Spitzen-Popularitätswerte für Joschka Fischer sind dafür ein Indikator.

Die folgenden Ausführungen sind unvollständig. Sie konzentrieren sich auf die Fallbeispiele Balkan und Terrorismus/Afghanistan sowie den Aufbau sicherheits- und friedenspolitischer Fähigkeiten (Krisenprävention und Bundeswehrreform).

\section{Bühnenwechsel}

Beide Koalitionsparteien waren unzureichend auf die außenund sicherheitspolitische Regierungsverantwortung vorbereitet. Vor allem in der Militärfrage bestand keine Klarheit und Einigkeit in und zwischen den beiden Parteien. Die Bündnisgrünen hatten im Kontext der Balkankriege und in einem schmerzhaften Erfahrungs- und Lernprozess wohl schrittweise ihre Haltung zu Gewaltfreiheit und Militär geändert, so dass die Bundestagsfraktion erstmalig am 19. Juni 1998 einem Auslandseinsatz der Bundeswehr (SFOR) zustimmte. In ihrem Wahlprogramm hatten sie aber noch wenig Rücksicht auf die Realitäten von Regierungsverantwortung genommen. Damit waren ab Koalitionsbeginn abrupte politische Schwenks einerseits und tiefe Enttäuschungen andererseits regelrecht vorprogrammiert.

Schon die Koalitionsvereinbarung verlangte Bekenntnisse zur NATO, zur Werte- und Interessengemeinschaft mit den USA und zur Bundeswehr, die der Einstellung vieler Grüner zuwiderliefen. Sehr schnell bekamen die Regierungsgrünen zu spüren, dass der Wechsel von der Opposition in die Regierung nicht ein Rollen-, sondern ein kompletter Bühnenwechsel war: Der »Spielplan« ist zu erheblichen Teilen fremdbestimmt; deutsche Außenpolitik agiert immer im multilateralen 
Kontext, nie im Alleingang; die internationalen Erwartungen (und Befürchtungen) gegenüber der deutschen Rolle und Verantwortung sind ganz andere als vor zehn Jahren; Regierende können sich nicht mehr mit der Kritik begangener Fehler und der Forderung des Wünschenswerten begnügen, sondern sind im Hier und Jetzt unter gegebenen Bedingungen und angesichts realer Handlungsalternativen gefordert - in Verantwortung für die Konsequenzen des eigenen Tuns oder Unterlassens. Vor allem bei internationalen Krisen waren Entscheidungssituationen unausweichlich, standen keine langen Bänke und Kommissionen zur Verfügung.

Diese unterschiedlichen Handlungsbedingungen von Regierung, Opposition und sozialen Bewegungen wurden von Rot-Grün zu wenig verständlich gemacht und von Kritikern - vor allem Friedensbewegten - kaum wahrgenommen. Dementsprechend oft reden Regierungsgrüne und Friedensbewegte mit falschen Erwartungen aneinander vorbei.

\section{Grundorientierungen}

Krieg ist und bleibt eine Geißel der Menschheit. Kriegsverhütung, die Überwindung der Institution des Krieges und Friedensförderung bleiben Kern grüner Außen- und Sicherheitspolitik. Angesichts der Dominanz innerstaatlicher Kriege, des Anwachsens privater Gewalt in schwachen und zerfallenden Staaten und des Leidens der Zivilbevölkerung dabei stellt sich die Frage, was die Internationale Gemeinschaft gegen diese realen bzw. akut drohenden Kriege und Massenvertreibungen bis zum Völkermord, gegen Gewaltakteure tun kann und muss. Sarajevo, Srebrenica, Ruanda und der andauernde Krieg im Kongo sind Extrembeispiele für die Handlungsschwäche der Staatengemeinschaft gegenüber der Herausforderung, akute Kriegsgewalt einzudämmen und zu beenden.

Gewaltfreiheit ist ein Grundwert der Grünen. Er muss in Regierungsverantwortung aber anders buchstabiert werden. Wer für den Schutz der eigenen Bevölkerung und für das staatliche Gewaltmonopol verantwortlich ist, kann nicht gleichzeitig prinzipielle Gewaltfreiheit praktizieren. In Regierungsverantwortung muss es darum gehen, durch Gewaltverhütung und Gewalteindämmung mehr Gewaltfreiheit $\mathrm{zu}$ schaffen und dabei die Mittel des rechtsstaatlichen Gewaltmonopols nur begrenzt und verhältnismäßig einzusetzen.

Militärkritik bleibt berechtigt und notwendig. Krieg und Militär lösen keine Konflikte. Einsatz von militärischer Gewalt bleibt ein Übel und tückisches Mittel. Aufrüstung fördert Aufrüstung und frisst Ressourcen, die einer menschlichen Entwicklung fehlen. Militär wurde und wird meistens im Kontext von Machtinteressen eingesetzt. Zugleich vollzog sich in den letzten Jahrzehnten ein teilweiser Funktionswandel von Militär: Im Rahmen des VN-Systems und kollektiver Sicherheitssysteme entwickelte sich eine ganze Spannweite von Militäreinsätzen und -aktivitäten von der Katastrophenhilfe über die Mitwirkung bei Rüstungskontrolle und Abrüstung sowie Dialog und Kooperation bis zu Einsätzen zur Friedenssicherung und Zwangsmaßnahmen gegenüber Bedrohungen der internationalen Sicherheit und des Weltfriedens. Solche multinationalen und begrenzten Militäreinsätze haben einen zunehmend polizeilichen und gewalteindämmenden
Charakter. Sie unterscheiden sich grundlegend von den Schlachten des Zweiten Weltkrieges oder imperialistischen Eroberungsfeldzügen.

Die Haltung der Grünen zu Krieg, Gewaltfreiheit und Militär differenzierte sich in Reaktion auf die aktuellen politischen Konflikte und fand ihren Niederschlag im Grundsatzprogramm von 2002.

\section{Herausforderung Balkan}

Als Rot-Grün »an die Macht« kam, war die Konflikteskalation im Kosovo schon weit fortgeschritten. Ausschlaggebendes Motiv der Koalition war, im Kosovo ein »zweites Bosnien « zu verhindern. Deutsches Drängen auf eine Verhandlungslösung blieb letztlich erfolglos. In dem Dilemma von Wertekonflikt und völkerrechtlicher Regelungslücke trug Rot-Grün die Luftangriffe auf die Bundesrepublik Jugoslawien mit. Es war dann Außenminister Fischer, der während der NATO-Luftangriffe einen Friedensplan entwickelte, bei dem Russland und die Vereinten Nationen wieder »ins Spiel« kamen und der zur Beendigung des Krieges knapp vor einer umfassenden Eskalation beitrug.

Bis heute ist der Tabubruch der ersten bundesdeutschen Kriegsbeteiligung umstritten. Eine öffentliche selbstkritische Aufarbeitung des Kosovo-Krieges, seiner Wirkungen, Kosten und Opfer wurde wohl von einzelnen Koalitionspolitikern gefordert und betrieben, von der eigenen Regierung aber weitgehend gemieden. Nichtsdestoweniger wurden entscheidende Konsequenzen aus dem gezogen, was für uns kein Präzedenzfall, sondern abschreckendes Beispiel war.

Die Europäische Sicherheits- und Verteidigungspolitik bekam einen starken Schub - anfänglich mit einer kurzsichtigen militärischen Schlagseite, dann aber auch durch neue Mechanismen nichtmilitärischer Krisenbewältigung.

Was die Grünen vor Jahren im Bundestag erfolglos gefordert hatten, wurde jetzt umgesetzt. Die Bundesrepublik wurde zum Vorreiter des Balkan-Stabilitätspaktes, mit dem die Staatengemeinschaft erstmalig für eine ganze Krisenregion ein umfassendes und kooperatives Stabilisierungsprogramm auflegte. Endlich wurde die demokratische Opposition in Serbien massiv unterstützt. Diese »zivile Intervention« trug zur friedlichen Oktoberrevolution in Belgrad bei. In Montenegro, Südostserbien, Mazedonien drohten erneute Gewalteskalationen mit verheerenden Auswirkungen. Die Bundesrepublik war maßgeblich an den Bemühungen der Staatengemeinschaft beteiligt, diese Kriegsgefahren unter Kontrolle zu halten. In konzertierter Aktion zwischen EU, KFOR/NATO und neuer serbischer Führung gelang das im Presevotal. Die Öffentlichkeit nahm diesen Erfolg kaum zur Kenntnis.

Als in Mazedonien albanisch-stämmige Extremisten die Gefahr eines Bürgerkrieges heraufbeschworen, griff die Staatengemeinschaft unter Federführung der EU so früh, kohärent und konsequent ein wie bei keiner Balkankrise zuvor. Mit massiven positiven Angeboten und Druck wurden die Konfliktparteien zu einer politischen Konfliktlösung gedrängt. Die - anfangs umstrittene - NATO-Truppe unterstützte die politische Deeskalation durch Entwaffnungshilfe und den Schutz internationaler Beobachter. Der Frieden ist in 
Mazedonien noch längst nicht gewonnen, der Konflikt bleibt noch gewaltträchtig. Aber ohne die politische Intervention der Staatengemeinschaft wäre Mazedonien schon seit einem Jahr im Bürgerkrieg explodiert, wäre der ganze Balkan ins Rutschen gekommen. Das ist ein enormer, wenn auch unsichtbarer Erfolg europäischer Sicherheits- und Friedenspolitik.

\section{Herausforderung internationaler Terrorismus}

Mit den Terrorangriffen vom 11. September war Rot-Grün erstmalig akut mit der staatlichen Grundverantwortung für den Schutz der eigenen Bevölkerung, den Schutz der internationalen Sicherheit und den Schutz der offenen Gesellschaft konfrontiert.

Die bisher eher verdrängte Gefahr eines massenmörderischen Terrorismus war nun unübersehbar. Befürchtet werden musste eine Fortsetzung, ja Steigerung der Terrorbedrohung - vor allem im Hinblick auf den drohenden Zugriff hochorganisierter und opferbereiter Terrornetzwerke auf Massenvernichtungswaffen.

Für Rot-Grün gehörten von Anfang an direkte Täterverfolgung, Gefahrenabwehr und längerfristige Ursachenbekämpfung untrennbar zusammen. Erforderlich war das ganze Spektrum an diplomatischen, polizeilichen, geheimdienstlichen, finanz-, entwicklungs- und kulturpolitischen etc. Maßnahmen. Klar war, dass das Problem des transnationalen Terrorismus nicht militärisch gelöst, aber auch nicht ohne Militär eingedämmt werden kann. Zu offenkundig war die Schlüsselrolle Afghanistans als Ausbildungs- und Rückzugsgebiet des transnationalen Terrorismus und die Verquickung von Taliban-Regime und Al Qaida. In dieses hochmilitarisierte Umfeld polizeiliche Spezialkräfte zu schicken, wäre völlig aussichts- und verantwortungslos gewesen.

Der Bundeskanzler setzte das Wort von der »uneingeschränkten Solidarität«. Das klang wie »bedingungslose Solidarität« und stieß zu Recht auf Ablehnung. Das große Wort verdeckte die andere Realität. Die Bundesrepublik beteiligte sich an der Bekämpfung des internationalen Terrorismus von Anfang an mit selbstbewusster Solidarität: primär politisch, unbedingt multilateral, begrenzt auch militärisch. Die Masse der militärischen Beiträge sind Unterstützungsleistungen (Transport, ABC-Schutz, Seeraumüberwachung). Bis zu hundert Spezialsoldaten sind an der direkten Bekämpfung von Al Qaida beteiligt. Der Bundestag hat dazu eindeutig klargestellt, dass die militärische Bekämpfung dieses Terrornetzwerkes und seiner Unterstützer an die Regeln des (Kriegs-)Völkerrechts gebunden ist.

Seit September 2001 ist Deutschland führend an der Stabilisierung Afghanistans nach 23 Jahren Kriegschaos beteiligt. Nicht von ungefähr fand die zentrale Afghanistan-Konferenz (Petersberg, Humanitäre Hilfe, Förderung der Zivilgesellschaft, Polizeiaufbau) in Deutschland statt. Die Bundeswehr stellt ca. $20 \%$ der internationalen Schutztruppe ISAF in Kabul und hat seit März 2002 die operative Führung der multinationalen Brigade. In Kabul und Umgebung bewirkte ISAF bisher ein relativ sicheres Umfeld für Hilfe, Wiederaufbau, Regierung und öffentliches Leben. Ende 2001 drohte in Afghanistan eine riesige humanitäre Katastrophe. Diese konnte die Internationale Gemeinschaft in konzertierter
Aktion abwenden. Der Umfang der Flüchtlingsrückkehr übertrifft alle Erwartungen.

Die Schwerpunkte des deutschen Engagements liegen in der Humanitären Hilfe, im Minenräumen, in der Basisgesundheitsversorgung, im Schul- und Bildungswesen, in der Frauenförderung, der Förderung von Zivilgesellschaft und Rechtsstaatlichkeit. Die Gesellschaft für Technische Zusammenarbeit organisierte die Logistik der Großen Ratsversammlung.

Angesichts der vielen tausend Bewaffneten, der grassierenden Fraktionskämpfe und Kriminalität und der Unmöglichkeit einer flächendeckenden ISAF-Stationierung ist der Aufbau afghanischer Sicherheitsstrukturen von strategischer Bedeutung für die Befriedung des Landes. Die Bundesrepublik hat die Führungsverantwortung beim Aufbau der afghanischen Polizei übernommen.

Die Befürchtung vom November 2001, die Bundesrepublik beteilige sich an einem »Krieg gegen Afghanistan « und gerate in ein afghanisch-amerikanisches Kriegsabenteuer, hat sich nicht bewahrheitet. Deutschland unterstützt vor allem Kriegseindämmung und nation building in Afghanistan.

Ausschlaggebend für das deutsche Engagement auf dem Balkan, in Afghanistan und am Horn von Afrika sind nicht machtpolitische oder gar imperialistische Interessen, wie es PDS oder Teile der Friedensbewegung unterstellen. Maßgeblich ist vielmehr das deutsche Sicherheitsinteresse an multilateraler Krisenprävention, Kriegsverhütung und Stabilisierung auf dem Balkan und an einer wirksamen Bekämpfung des transnationalen Terrorismus, seiner Rückzugsräume und Nährböden. Dabei reduziert sich das deutsche Engagement keineswegs auf reaktive Militäreinsätze, wie es in einer militärfixierten Öffentlichkeit, aber auch bei genauso militärfixierten linken Kritikern den Anschein hat. Es ist vorrangig politisch und auf umfassende und vorbeugende Sicherheit orientiert. Die Förderung von Zivilgesellschaft und legitimer Staatlichkeit gegenüber privater Gewalt hat einen immer höheren Stellenwert. Bei Friedensmissionen haben die zivilen Komponenten ein immer stärkeres Gewicht.

Zugleich wächst die Kluft zwischen dem deutschen und europäischen Ansatz der primär politischen Terrorismusbekämpfung und dem der USA. Die Bush-Regierung instrumentalisiert den Kampf gegen den Terror zusehends für innenpolitische und Hegemonialinteressen. Sie betreibt ihn in erster Linie militärisch, unilateral und mit einem Kooperationsmuster, wo sich willige »Juniorpartner« wie Speichen um die »Radnabe« USA gruppieren. Ihr »Krieg gegen den Terror « überschreitet bisherige Grenzen und droht, zu einem globalen Dauerkrieg zu werden.

\section{Strukturelle Krisenprävention}

Krisenprävention hat eine langfristig-strukturelle und eine kurzfristigere operative Dimension. Aktuelle Berichte der Bundesregierung über ihre Menschenrechtspolitik (Juni 2002, BT-Drs.14/9323), zum Stand der Bemühungen um Abrüstung (April 2002, BT-Drs.14/8941), zur Auswärtigen Kulturpolitik (Juli 2002, BT-Drs.14/9760), zur Entwicklungspolitik (2001, BT-Drs.14/6496) und vor allem der erstmalige Bericht zur 
Zusammenarbeit der Bundesrepublik Deutschland mit den Vereinten Nationen (Juni 2002, BT-Drs.14/9466) geben dazu einen umfassenden und detaillierten Überblick.

Im Sinne der langfristigen Krisenprävention engagiert sich Rot-Grün besonders für

- die europäische Integration und Erweiterung als das zentrale Friedensprojekt auf dem früheren »Kontinent der Kriege «,

- den Aufbau des Internationalen Strafgerichtshofes (Verabschiedung des deutschen Völkerstrafgesetzbuches) als zentrale Säule einer globalen Rechtsordnung und die umfangreiche Unterstützung der Internationalen Strafgerichtshöfe für Ex-Jugoslawien und Ruanda;

- die Stärkung der Menschenrechte (Aufwertung des Unterausschusses Menschenrechte und Humanitäre Hilfe zum Vollausschuss des Bundestages, Gründung eines Menschenrechtsinstitutes, Anerkennung nichtstaatlicher und geschlechtsspezifischer Verfolgung im neuen Zuwanderungsgesetz),

- die Stärkung der Vereinten Nationen und der OSZE (Reform der VN-Missionen, stand-by-Abkommen, Initiative zur Effektivierung des Sanktionsinstrumentariums, Ausbau Bonns als VN-Stadt);

- den internationalen Klima- und Ressourcenschutzes (z. B. Internationale Wasserkonferenz in Bonn). Wo der Zugang zum Öl und anderen Ressourcen eine häufige Konfliktund Kriegsursache ist, da ist die unter Rot-Grün begonnene Energiewende hin zu den erneuerbaren Energien strategische Friedenspolitik par excellence!

- die Aufwertung der Entwicklungspolitik, die erstmals als politische Querschnittaufgabe verankert wurde (Kompetenzerweiterung des BMZ, Mitgliedschaft im Bundessicherheitsrat, entwicklungspolitische Regelprüfung von Gesetzesvorhaben), und den systematischen Ausbau umweltrelevanter Programme; vermehrte Orientierung auf Friedensentwicklung (Förderung von Friedensursachen). Angesichts der notwendigen Haushaltskonsolidierung konnte erst 2002 wieder ein Anstieg des Entwicklungshaushaltes erreicht werden. Manche nicht entwicklungsverträglichen Hermes-Bürgschaften und Rüstungsexportentscheidungen wurden allerdings mit »höchstem Segen« gegen den Widerstand der Grünen durchgesetzt.

Rüstungskontrolle und Abrüstung befinden sich in der Krise. Die Bewahrung und Weiterentwicklung multilateraler Rüstungskontrolle wurde angesichts der Deregulierungspolitik der USA besonders schwierig. Die Gesamtbilanz ist nicht zufrieden stellend. Nichtsdestoweniger gab es Einzelerfolge: Anfang 2002 trat endlich der Vertrag über den Offenen Himmel in Kraft, der von Vancouver bis Wladiwostok Vertrauensbildung in beispiellosem Umfang ermöglicht. Damit die Bundesrepublik weiterhin vorbildlich zur Umsetzung und Weiterentwicklung des »Offenen Himmels« im Rahmen der Krisenprävention beitragen kann, soll eine europäische Beobachtungsplattform beschafft werden. Vorreiter ist die Bundesrepublik auch bei der Vernichtung von Chemiekampfstoffen in Russland im Rahmen der Abrüstungszusammenarbeit: Die erste, mit deutscher Hilfe errichtete C-Vernichtungsanlage nimmt in diesem Jahr ihren Betrieb auf. Auf deutsche Initiative beschloss die EU eine »Gemeinsame Aktion zur Bekämpfung der destabilisierenden Anhäufung und Verbreitung von Kleinwaffen«. Deutschland und die EU-Partner brachten auf der VN-Kleinwaffenkonferenz den Entwurf eines Aktionsplanes ein. Das AA und das BMZ unterstützen weltweit verschiedene Demilitarisierungs- und Demobilisierungsprojekte. Im Rahmen des Stabilitätspaktes wurden in Albanien insgesamt 100.000 Kleinwaffen zerstört. Das Verteidigungsministerium hat angekündigt, in den kommenden Jahren 400.000 ausgemusterte G-3-Gewehre der Bundeswehr zu zerstören. Der Bundestag beschloss einen Koalitionsantrag zur »Weiterentwicklung der humanitären Rüstungskontrolle bei Landminen«, der wichtige grüne Positionen zu nationalen Vorleistungen beinhaltet. Der ebenfalls von SPD und Grünen initiierte Bundestagsbeschluss zur kooperativen Rüstungskontroll- und Abrüstungspolitik (BT-Drs.14/9241) verdeutlicht die Alternative zum gegenwärtigen destruktiven US-Kurs.

Die Eindämmung des Rüstungsexports in Krisenregionen oder Staaten, die die Menschenrechte missachten, ist unerlässlich für eine glaubwürdige und wirksame Krisenprävention. Hier konnten die Bündnisgrünen mit der Überarbeitung der Exportrichtlinien einen wichtigen Teilerfolg erringen, der in der Regierungspraxis aber immer wieder neu erstritten werden muss. Mit den Richtlinien wird die Exportpolitik der Bundesrepublik transparenter und damit kontrollierbarer. Die Grünen stehen im ständigen Konflikt mit massiven Interessen, die versuchen, die bisherigen Restriktionen zu unterlaufen und zu lockern.

\section{Infrastruktur zivile Konfliktbearbeitung}

Zur Stärkung der kurzfristigen operativen Krisenprävention versprach der rot-grüne Koalitionsvertrag den Aufbau einer Infrastruktur Krisenprävention und zivile Konfliktbearbeitung. Das Auswärtige Amt und das Ministerium für wirtschaftliche Zusammenarbeit und Entwicklung begannen sofort damit. Die deutsche Beteiligung am Kosovo-Krieg machte das Vorhaben nicht zur Makulatur, sondern bekräftigt viel mehr seine Dringlichkeit. Der Bundessicherheitsrat beschloss im April 2000 ein »Gesamtkonzept Krisenprävention und Konfliktbeilegung « und betonte dabei vor allem den multidimensionalen, kooperativen Ansatz.

In der Entwicklungszusammenarbeit wurde die Dimension der Krisenprävention in mehrfacher Hinsicht gestärkt. Sie wurde einbezogen in Länderkonzepte und Schwerpunktstrategien. Zur Konfliktsensibilisierung finden Qualifizierungskurse für Mitarbeiter verschiedener Bereiche statt.

Das BMZ fördert den Aufbau des Zivilen Friedensdienstes in der bilateralen Entwicklungszusammenarbeit durch Mitfanzierung der Ausbildung Zivile Konfliktbearbeitung und Unterstützung von inzwischen 38 Projekten in 32 Ländern. Der ZFD soll Friedenspotenziale mit lokalen Partnern stärken, bei Konflikten zwischen Angehörigen von Interessengruppen, Ethnien und Religionsgruppen vermitteln, zu Versöhnung und Wiederaufbau beitragen.

Bis Anfang 2002 wurden 31 Mio. $€$ für die mehrjährigen Einsätze von 121 Friedensfachkräften zur Verfügung gestellt. Inzwischen ist die zweijährige Aufbauphase abgeschlossen. Die Evaluierung ergab eine sehr positive Gesamtbewertung. 
Die Gesellschaft für Technische Zusammenarbeit/GTZ richtete ein Sektorberatungsvorhaben Krisenprävention und Konfliktbearbeitung ein. Hier wird u. a. die rechtsstaatliche Reform des Sicherheitssektors unterstützt.

Seit Sommer 1999 führt das AA Kurse durch, in denen zivile Experten auf ihre Arbeit in internationalen Friedensmissionen von VN und OSZE vorbereitet werden. Inzwischen wurden die Kurse international geöffnet. Der Pool für ziviles Friedenspersonal umfasst bisher mehr als 700 Personen und wächst kontinuierlich. Um die Rekrutierung und Qualifizierung des Friedenspersonals zu verbessern, ihre Einsatzbegleitung und Nachbetreuung sowie die Evaluation von Friedenseinsätzen zu gewährleisten, wurde am 24. Juni 2002 das »Zentrum für Internationale Friedenseinsätze «/ZIF mit ca. 20 MitarbeiterInnen in Berlin eröffnet. Mit dem ZIF ist die Bundesrepublik auf gutem Wege, weltweit neue Akzente zu setzen.

Nichtmilitärische Polizeikräfte (CIVPOL) spielen bei internationalen Friedensmissionen in beratender, beobachtender und auch exekutiver Funktion eine immer bedeutendere Rolle. Die Bundesrepublik leistet dazu mit zurzeit ca. 550 BeamtInnen von BGS und Länderpolizeien auf dem Balkan sowie in Afghanistan (s.o.) einen quantitativ und qualitativ vorbildlichen Beitrag. Angesichts des EU-Beschlusses, bis 20035.000 Polizisten für Friedensmissionen zur Verfügung stellen zu können, bereiten sich Bund und Länder auf die Bereitstellung und Qualifizierung des deutschen 20\%-Anteils vor.

Die Bundesrepublik ist an fünf von $14 \mathrm{VN}-$ Missionen personell vor allem mit Polizisten und Zivilexperten beteiligt. Bei den VN-mandatierten Missionen in Bosnien-Herzegowina, Kosovo, Mazedonien und Kabul gehört sie zu den größten Truppenstellern.

Seit 2000 fördert das AA mit einem - auf grüne Initiative auf 14,5 Mio. $€$ erhöhten Etatansatz umfassend Maßnahmen der Krisenprävention und des Peacebuilding der VN, von Regionalorganisationen und erstmals auch von Nichtregierungsorganisationen. Die Projekte reichen von Menschenrechtsschutz in Kolumbien über Integrationsprozesse mit der russischen Minderheit im Baltikum und der Förderung von interethnischen Dialogprozessen bis zu Entwaffnungs-, Demobilisierungs- und Reintegrationsprogrammen.

Im April 2001 wurde unter Federführung des Bundesforschungsministeriums die Deutsche Stiftung Friedensforschung (DSF) mit Sitz in Osnabrück gegründet und damit die Bundesförderung einer unabhängigen Friedens- und Konfliktforschung wieder aufgenommen. Ihr Grundkapital beträgt 25 Mio. $€$. Sie finanziert Forschungsprojekte, einen geistes- und sozialwissenschaftlichen Studiengang, einen PostgraduiertenStudiengang »Friedensforschung und Sicherheitspolitik« sowie Promotionsstipendien.

Die nationalen Maßnahmen würden wirkungslos versickern, wenn sie nicht einhergingen mit Bemühungen auf multilateraler Ebene, die Fähigkeiten der Gewalt- und Krisenprävention zu stärken. Zusammen mit den skandinavischen Ländern gehört hier die Bundesrepublik zu den treibenden Kräften. Auf internationaler Ebene ist Krisen- und Gewaltprävention inzwischen $\mathrm{zu}$ einem Top-Thema avanciert und steht nun auch vorne auf der Agenda von EU, G-8 und NATO. Die OSZE beschloss im Juni 2000 das REACT-Programm (Rapid
Expert Assistance and Cooperation Teams) zur kurzfristigen Mobilisierung von zivilen Fachleuten für OSZE-Missionen.

Am deutlichsten sind die Fortschritte im Rahmen der Weiterentwicklung der Europäischen Sicherheits- und Verteidigungspolitik, auch wenn das Hauptaugenmerk von Politik und Öffentlichkeit auf der geplanten Eingreiftruppe liegt. Der EU-Gipfel von Helsinki beschloss im Dezember 1999, einen Mechanismus nichtmilitärischer Krisenbewältigung aufzubauen. Erste Planziele wurden beschlossen für internationale nichtmilitärische Polizeieinsätze (s.o.). Weitere in $2003 \mathrm{zu}$ erreichende Planziele sind inzwischen aufgestellt für die Bereitstellung von Fachpersonal zur Stärkung von Rechtsstaatlichkeit (200), der Zivilverwaltung (Pool) und des Katastrophenschutzes (2.000). Der EU-Gipfel von Göteborg beschloss ein säulenübergreifendes EU-Programm »zur Verhinderung gewaltsamer Konflikte«, in dem der Krisenfrüherkennung und gemeinsamen Übungen von Kriseneinsätzen besondere Bedeutung beigemessen wird. Die Instrumente nichtmilitärischer Krisenbewältigung werden eher einsatzbereit sein als die militärischen Kräfte der EU.

\section{Aktionsplan Krisenprävention}

Die Jahre seit 1999 markieren einen Durchbruch beim Aufbau von Fähigkeiten nichtmilitärischer Gewalt- und Krisenprävention auf nationaler und internationaler Ebene. Hier kamen die Grünen erheblich voran bei der Umsetzung und Operationalisierung des Grundwertes Gewaltfreiheit. Diese »Programmtreue« war keineswegs eine Alibiveranstaltung, sondern hat eigenes Gewicht und eigene Dynamik. Die bisher eingeleiteten Maßnahmen sind ein guter Start und haben die Fähigkeit der Bundesrepublik, zur internationalen Krisenbewältigung beizutragen, deutlich verbessert. Um aber den Rückstand gegenüber den Fähigkeiten militärischer Krisenbewältigung aufzuholen und eine wirksame Krisenprävention aufzubauen, sind erhebliche weitere Anstrengungen unabdingbar. Dazu gehören u. a.:

- Aufbau angemessener und integrierter Analyse- und Frühwarnungskapazitäten auf staatlicher und nichtstaatlicher Ebene. Das setzt eine bessere Personalausstattung des Auswärtigen Amtes wie eine Aufstockung der Fördermittel für Friedensforschung voraus.

- Förderung der Bereitschaft und Fähigkeiten zur Teilnahme an internationalen Friedenseinsätzen und Friedensarbeit durch Studien- und Ausbildungsgänge, Attraktivitätssteigerungen, Einsatzbegleitung und Wiedereingliederungshilfen.

- Förderung der schnellen Verfügbarkeit von Friedenspersonal durch Freistellungsregelungen mit öffentlichen und privaten Arbeitgebern.

- Stärkung von Kooperation und Integration bei allen Kräften, die zur Friedenssicherung und Gewaltprävention beitragen sollen.

- Um den zugesagten deutschen Beitrag von bis zu 1.000 Beamten zu internationalen Polizeimissionen erfüllen und über längere Zeiträume durchhalten zu können (Rotationsfaktor 3, also bis zu 3.000 Beamte), ist eine Personalaufstockung bei Bund und Ländern unumgänglich. Das muss einhergehen mit einer Weiterentwicklung ihrer Ausbildung. 
- Damit der Zivile Friedensdienst über die Graswurzelebene hinaus stärker regional wirksam werden kann, sollte in den nächsten vier Jahren das Potenzial an Friedensfachkräften vervielfacht werden. Das muss einhergehen mit einer schrittweisen Steigerung der Projektförderung (Größenordnung 50 Mio. €).

- Krisenprävention und zivile Konfliktbearbeitung sind medial besonders schwer vermittelbar. Umso notwendiger ist, sie mit professionellen Methoden bekannt und verständlich $\mathrm{zu}$ machen und so $\mathrm{zu}$ einer »Alphabetisierung « in ziviler Konfliktbearbeitung beizutragen.

- In einem »Aktionsplan Krisenprävention« sind die entsprechenden Aufgabenfelder und die dafür notwendigen Fähigkeiten (mit Planzielen) festzulegen. Die Friedensinvestitionen müssen stetig anwachsen.

\section{Bundeswehrreform}

Auf dem Feld der Militärpolitik klafften grüne - und friedensbewegte - Wünsche und Wirklichkeit besonders weit auseinander. Forderungen nach Auflösung der Krisenreaktionskräfte etc. waren von vorneherein für die SPD nicht verhandelbar. Die relative Radikalität des grünen Programms von 1998 schwächte die Verhandlungsposition der Grünen statt sie zu stärken. Die verbliebene Friedensbewegung brachte kein zusätzliches politisches Gewicht auf die Waagschale, weil ihre Abrüstungsforderungen noch weiter von dem auf Bundesebene Diskutablen entfernt waren und konstruktive Vorschläge weitgehend fehlten.

Die bundesdeutsche Sicherheitspolitik leidet seit Jahren unter einem Demokratiedefizit. Eine umfassende gesellschaftliche Debatte und Verständigung über Rolle, Leistungsfähigkeit und Grenzen von Militär in der deutschen Außen- und Sicherheitspolitik nach Ende des Ost-West-Konflikts wurde gemieden, unter Rühe wie unter Scharping. Die Chance, in der entscheidenden Umbruchphase der Bundeswehr die Menschen angesichts neuer Herausforderungen »mitzunehmen«, wurde vertan.

Der gründliche Bericht der Weizsäcker-Kommission zur Bundeswehrreform wurde schnell ausmanövriert. Schon drei Wochen später setzte Minister Scharping die von ihm vorgelegten »Eckpfeiler « zur Reform der Bundeswehr im Bundeskabinett durch. Ihre Intention, die Bundeswehr besser auf Krisenbewältigung auszurichten und $\mathrm{zu}$ modernisieren, war schon angesichts des mit KFOR erheblich ausgeweiteten Balkaneinsatzes richtig. Die Eckpfeiler blieben aber deutlich hinter den Empfehlungen der Kommission zurück, sie waren angesichts der veränderten sicherheitspolitischen Lage und der begrenzten Haushaltsmittel nicht konsequent genug. Wider massivsten Erhöhungsforderungen konnte der Konsolidierungskurs im Wesentlichen auch beim Militärhaushalt gehalten werden. Der Ressortminister setzte die Bundeswehrreform ohne koalitionsinterne Konsensbildung faktisch per Dekret in Kraft. Der Alleingang hielt an bis zu seiner Entlassung.

Die Grünen betonten demgegenüber ihre eigenständige Position mit in einem Konzept zur Bundeswehrreform, das zumindest gute Resonanz bei Friedensforschern wie bei aufgeschlossenen Militärs fand.
Die Angebote des »Friedenswortes« der katholischen Bischöfe und der EKD-Denkschrift zu einer grundlegenden friedensethischen Verständigung wurden von Politik und Öffentlichkeit nicht aufgenommen. Statt dessen entwickelt sich deutsche Sicherheitspolitik durch exekutives Handeln und Fall-zu-Fall-Entscheidungen, nach dem 11. September mit einem regelrechten Quantensprung. Insbesondere die Grünen setzten sich dabei für die militärische Beschränkung und Ausgewogenheit des deutschen Beitrags ein. Die Bundestagsentscheidungen zum Mazedonien- und Anti-Terror-Einsatz der Bundeswehr wurden zu einer äußersten Belastung der gesamten Koalition. Statt die verbreiteten Ängste vor einem »rot-grünen Vietnam《 in Afghanistan überzeugend zu entkräften, setzte sich der Kanzler mit Hilfe des sachfremden Machtworts der Vertrauensfrage durch.

\section{Strategiemangel}

Soweit die Bundeswehrreform in der Öffentlichkeit und im Bundestag Thema war, dominierten die Sekundärfragen des WIE: Modernisierung, Haushaltsfragen, Wehrform. Die Primärfragen des WOFÜR und WOGEGEN wurden regelmäßig vernachlässigt.

Die Planungsdokumente der Bundeswehrreform begnügten sich damit, die auf NATO- und EU-Ebene zwischen den Regierungen vereinbarten Aufträge, Aufgaben und Fähigkeiten als Vorgaben zu benennen. Ihre Ableitung aus einer differenzierten Risiko- und Bedrohungsanalyse liegt bisher nicht vor. Das »Weißbuch« des Bundesministers der Verteidigung, das dies leisten müsste und seit langem angekündigt ist, ist immer noch nicht erschienen.

Eine umfassende und existentielle Bedrohung des Territoriums der Bundesrepublik bzw. des Bündnisgebiets durch eine raumgreifende Aggression, das Risiko des »großen Verteidigungsfalls« ist auf mittlere Frist nicht nur unwahrscheinlich, sondern auszuschließen. Die Bundeswehr als Massenarmee zur Abwehr von gepanzerten Angriffen auf das Bundesgebiet gehört der Vergangenheit an. Andere militärische Risiken und potentielle Bedrohungen hingegen sind mit unterschiedlicher Eintrittswahrscheinlichkeit real: Auswirkungen und Ausweitungen von Regionalkonflikten, Terrorismus mit konventionellen und ABC-Massenvernichtungsmitteln, mittelfristig auch Raketenangriffe, cyberwar-Attacken, Globalisierung privater Gewalt. Die Krisenherde von Mazedonien, des ganzen Nahen und Mittleren Ostens, die ordnungslosen Räume zerfallener Staaten beinhalten Risiken, die massiv die Sicherheit der Bundesrepublik und des Bündnisses betreffen und die internationale Sicherheit und den Weltfrieden gefährden können. Seit dem 11. September ist auch das Undenkbare möglich geworden.

Die Komplexität der Sicherheitspolitik ist zu einer eigenständigen Herausforderung geworden: Konfliktursachen können sich weiträumiger entwickeln; große Veränderungen geschehen in kurzen Zeiträumen; Risiken werden vielfältiger; die steigende Zahl an Mitwirkenden verkomplizieren die internationalen Beziehungen. 


\section{Anforderungen und Auftrag}

Um diese Risiken abzuhalten, einzudämmen und abzuwehren, ist eine Politik erforderlich, die auf umfassende, vorbeugende und gemeinsame Sicherheit und gerechten Frieden zielt. Dabei geht es neben der Gefahrenabwehr und Ursachenbekämpfung vor allem auch darum, strategische Verwundbarkeiten der modernen Gesellschaft $\mathrm{zu}$ reduzieren, angefangen bei einer an Öl und Atom hängenden Energieversorgung.

Grundsätzliche Zweifel bleiben, ob eine hochindustrialisierte Gesellschaft wie die der Bundesrepublik überhaupt militärisch verteidigt $-\mathrm{d}$. h. bewahrt - werden könnte. Allerdings hat das Grundgesetz den Bund zur Aufstellung von Streitkräften zur Verteidigung verpflichtet.

Angesichts der NATO-Osterweiterung ist für die Bundesrepublik die Landesverteidigung völlig in der Bündnisverteidigung aufgegangen. Da auf mittlere Frist kein »großer« Verteidigungsbedarf besteht, können die dafür vorgehaltenen Kräfte und Aufwuchspotenziale erheblich reduziert werden.

Seit dem 11. September hat das herkömmliche Verständnis von Verteidigung eine enorme Entgrenzung erfahren. Der VN-Sicherheitsrat gab mit den einschlägigen Resolutionen, die das Recht auf individuelle und kollektive Selbstverteidigung bekräftigten, eine Art von Einstiegslegitimation für die militärische Bekämpfung des Terrorismus. Immer deutlicher stellt sich aber die Frage, wo die Grenzen dieses Selbstverteidigungsrechtes sind. Wenn die US-Regierung inzwischen das Recht für sich in Anspruch nimmt, gegebenenfalls mit Präemptionsangriffen zu jeder Zeit und an jedem Ort gegen terroristische Bedrohungen vorzugehen, dann wird damit das allgemeine Gewaltverbot der VN-Charta unterhöhlt und seine Beachtung in das Belieben der Stärksten gestellt.

Demgegenüber muss klar sein: Militärische Bekämpfung des internationalen Terrorismus muss Teil globaler Ordnungspolitik und Aufgabe von Systemen kollektiver Sicherheit sein. Nur hierüber erfährt sie völkerrechtliche Legalität und Begrenzung.

Kernauftrag der Bundeswehr ist, zur multilateralen Kriegsverhütung und-eindämmung im Dienste gemeinsamer Sicherheit im Rahmen des UN-Systems beizutragen. Diese ist nur aussichtsreich als multinationale und multidimensionale Politik im Verbund verschiedener staatlicher und gesellschaftlicher Akteure. Isolierte militärische Krisenbewältigung ist von vornherein zum Scheitern verurteilt. Ein besonderes Gewicht hat die Absicherung und Unterstützung von Friedensprozessen und die Stärkung rechtsstaatlicher Gewaltmonopole gegenüber privatisierter Gewalt (nation building).

Auch wenn Erzwingungseinsätze mit kriegerischer Gewalt »äußerst problematisch « und ein »tückisches Mittel « (Grundsatzprogramm) sind, so gebieten es doch die Vorgaben der VN-Charta und NATO, solche Kapazitäten zur Bündnisverteidigung und für Einsätze zur Sicherung bzw. Wiederherstellung des Weltfriedens vorzuhalten.

Durch Dialog und Kooperation sowie Beteiligung an Rüstungskontrolle und Abrüstung leistet die Bundeswehr hoch effektive und zugleich sehr kostengünstige Beiträge zur Vertrauensbildung und gemeinsamer Sicherheit.
Manche Äußerungen aus den rot-grünen Spitzen erwecken den Eindruck, als seien Militäreinsätze für die deutsche $\mathrm{Au}-$ ßen- und Sicherheitspolitik nun ein »normales« Instrument wie viele andere. Dem ist nicht so! Militäreinsätze sind ein besonders kostspieliges und riskantes Mittel. Deshalb gibt es den Parlamentsvorbehalt, deshalb werden Entsendeentscheidungen mit besonders hohem Verantwortungsbewusstsein gefällt.

Angesichts der Globalisierung von Unsicherheit, der europäischen Integration und des Unilateralismus der Hypermacht USA ist das Festhalten an nationalen Armeen in Europa ein teurer Anachronismus. Die schrittweise militärische Integration muss mit der Demokratisierung der EU einhergehen.

\section{Fähigkeiten und Strukturen}

Um ihren Auftrag verantwortlich im multinationalen Kontext durchführen zu können, braucht die Bundeswehr präsente und professionelle, flexible und hochmobile, insgesamt »leichtere « Kräfte mit hoher »Durchhaltefähigkeit « (Personalreserven, Logistik) und »Überlebensfähigkeit« (Selbstschutz). Sie setzen effektive Aufklärungs-, Führungs- und Verlegefähigkeiten auf große Distanz voraus. Die Vielfalt an Einsatzformen erfordert Soldaten mit breiten Fähigkeiten, insbesondere auch zur zivil-militärischen Zusammenarbeit. Das setzt eine qualitativ gute Nachwuchsgewinnung und sozialverträgliche Gestaltung von Auslandseinsätzen voraus. Wo wie derzeit 10.000 Soldaten im Auslandseinsatz sind, stehen Innere Führung und die Integration der Streitkräfte in die Gesellschaft vor erheblich veränderten Anforderungen.

Die Bundeswehr braucht nicht Alleskönner zu sein. Zunehmend mehr Fähigkeiten sollten gemeinsam mit europäischen Partnern betrieben werden. Neben dem Lufttransport und der strategischen Aufklärung stünde die Aufstellung einer integrierten Brigade für UN-Einsätze auf der Tagesordnung. Zugleich sollten zivile Aufgaben in Krisenregionen auch so weit und so schnell wie möglich von - in der Regel billigeren - zivilen Kräften übernommen werden.

Wo es entscheidend auf sich ergänzende militärische, polizeiliche und zivile Fähigkeiten ankommt, müssen endlich auch die Kräfteplanungen aufeinander abgestimmt werden. Ein Hinterherhinken der nichtmilitärischen Fähigkeiten verlängert nur die militärische Einsatzdauer.

Die Wehrpflicht ist sicherheitspolitisch nicht mehr legitimierbar und blockiert eine finanzierbare Bundeswehrreform.

Angesichts des Konsolidierungsgebots und des besonderen Nachholbedarfs des Außen- und Entwicklungsressorts ist die Begrenzung und Verstetigung des Militärhaushaltes unabdingbar. Eine Gesamtstärke in der Größenordnung von 200.000 Zeit- und Berufssoldatinnen und -soldaten reicht aus, um die im Rahmen von NATO und EU vereinbarten Beiträge sowie UN-Unterstützung leisten zu können.

\section{Einhegungen}

In einer Art sicherheits- und friedenspolitischer Selbstzufrie- 
denheit wird oftmals verdrängt, dass die Modernisierung der Bundeswehr ein zweischneidiges Schwert ist. Der Umbau ist erforderlich, um die auf Jahre notwendigen SFOR-, KFORund ISAF-Einsätze durchzuhalten. Er passt Fähigkeiten zur Bündnisverteidigung an heutige und künftige Bedingungen an.

Die Modernisierung schafft zugleich Interventionsfähigkeiten, die über kurz oder lang auch missbraucht werden können. Heute fühlen sich noch alle Bundestagsfraktionen, die Bundesregierung und die militärische Führung einer militärischen Zurückhaltung verpflichtet. Aber was ist in zwei, fünf Jahren wenn z. B. die ganze Division Spezielle Operationen und das ganze KSK einsatzfähig sind, wenn das gewachsene militärische Angebot die Nachfrage erhöhen kann?

Deshalb kommt es heute entscheidend darauf an, diese zweischneidigen Fähigkeiten politisch einzuhegen und an klare Kriterien zu binden. Hierzu machte ich vor Jahren Vorschläge, die inzwischen weitgehend in die grüne Programmatik eingingen:

- Bindung an die allgemeingültigen Werte des Völkerrechts und der Menschenrechte;

- Wahrnehmung kollektiver Sicherheitsinteressen, nicht von partikularen Interessen;

- Vorrang und Ausschöpfung nichtmilitärischer Krisenbewältigung;

- Bindung an ein Mandat der VN bzw. einer ihrer Regionalorganisationen;

- Auftragsklarheit und -begrenzung, keine Blankoschecks;

- Ziel und Grenzen des Einsatzes bedürfen der Zustimmung des Bundestages;

- Priorität des euroatlantischen Raumes;

- Einbindung in ein aussichtsreiches politisches Konzept;

- Ausreichende personelle und materielle Fähigkeiten;

- Verantwortbare Risiken und Kosten.

Diese Kriterien ziehen eine klare Trennungslinie zu einem - in Vergangenheit und Gegenwart reichlich praktizierten - machtpolitischen Interventionismus einerseits und einem potenziell schrankenlosen »humanitären Interventionismus « sowie einem »ewigen« Anti-Terror-Krieg andererseits.

Der Parlamentsvorbehalt ist nicht nur verfassungsrechtlich vorgeschrieben, sondern auch politisch überaus sinnvoll. Er gewährleistete bisher eine besonders intensive parlamentarische Beratung und trug zu breiter Konsensbildung bei. Der Parlamentsvorbehalt ist ein Eckstein der militärpolitischen Zurückhaltung der Bundesrepublik. Die Grünen fordern in ihren Programmen, Auslandseinsätze an eine Zweidrittelmehrheit des Bundestages zu binden. Dies ist vor allem bei solchen Einsätzen sinnvoll, die besonders riskant und umfangreich sind. Nach den Erfahrungen der letzten Jahre habe ich Zweifel, ob damit allein der gebotenen militärischen Zurückhaltung geholfen wäre.
Unzweifelhaft ist aber eine Konkretisierung des Parlamentsvorbehalts im Hinblick auf Spezialkräfte überfällig. Diese agieren im Kontext verdeckter und »unkonventioneller« Einsätze und damit schnell in einer völkerrechtlichen »Grauzone «. Der ministerielle Umgang mit den besonderen Geheimhaltungserfordernissen hat bisher eine parlamentarische Kontrolle durch den Verteidigungsausschuss unmöglich gemacht. Die unverzichtbare parlamentarische Kontrolle muss durch ein der Geheimdienstkontrolle vergleichbares Gremium gewährleistet werden.

\section{Stürmische Aussichten}

Rot-Grün war seit 1998 mit Herausforderungen konfrontiert, die wir uns vorher auch in schlimmen Träumen nicht gedacht hätten. Es gibt keinerlei Veranlassung zu der Annahme, die außen- und sicherheitspolitischen Entwicklungen würden sich beruhigen. Im Gegenteil. $\mathrm{Zu}$ den andauernden Risiken aus ungelösten Regionalkonflikten, Verbreitung von Massenvernichtungswaffen, transnationalem Terrorismus etc. kommt eine Art von US-Politik, die immer mehr zur Schwächung statt zur Stärkung der internationalen Sicherheit beiträgt und die viel beschworene westliche Wertegemeinschaft zu zerstören droht. Ihre neue Offensivstrategie bedeutet eine Art Privatisierung von Gewalt und Sicherheit »von oben«. Ihr militanter Kampf gegen den Internationalen Strafgerichtshof läuft dem Bemühen der Europäer um eine Globalisierung des Rechts diametral entgegen. Der beabsichtigte Angriff auf den Irak droht die ganze explosive Region zu entzünden. Zugleich wachsen europaweit mit rechtspopulistischen Bewegungen zentrifugale, die europäische Integration gefährdende Kräfte.

Angesichts dieser gefährlichen Herausforderungen braucht es ein einiges, handlungsfähiges und selbstbewusstes Europa, ist ein partnerschaftliches Gegengewicht zu den USA notwendiger denn je. Friedenspolitik braucht zugleich eine Friedensbewegung, die dialog- und bündnisfähig ist und in Gesellschaft und Politik Gehör findet.

Die Bundesrepublik unter Rot-Grün ist mit Außenminister Fischer Garant und treibende Kraft einer Politik des Multilateralismus, der europäischen Integration, der Krisenprävention, der umfassenden und gemeinsamen Sicherheit und als solche unter den Partnern wie in den Vereinten Nationen hoch angesehen. Um damit weiter voran zu kommen, brauchen wir mehr Kraft und Friedensinvestitionen.

Bisher spricht nichts dafür, dass eine Regierung Stoiber/Westerwelle (Möllemann) diesen Kurs so halten könnte und würde. In ruhigen Zeiten mag das nicht auffallen. In stürmischen Zeiten ist das hoch gefährlich. 